\title{
BROADBAND PHOTOCURRENT ENHANCEMENT IN LONGWAVE INFRARED QUANTUM DOT PHOTODETECTORS BY SUB-WAVELENGTH SURFACE GRATINGS
}

\author{
PUMINUN VASINAJINDAKAW, GUIRU GU and XUEJUN LU* \\ Department of Electrical and Computer Engineering \\ University of Massachusetts Lowell, One University Avenue \\ Lowell, MA 01854, USA \\ *xuejun_lu@uml.edu
}

Received 7 May 2012

Revised 18 February 2013

Accepted 18 February 2013

Published 26 April 2013

\begin{abstract}
We report a sub-wavelength transmission surface grating (TSG) enhanced longwave infrared (LWIR) quantum dot photodetector. Broadband photocurrent enhancement covering the wavelength range from $4 \mu \mathrm{m}$ to $11 \mu \mathrm{m}$ is observed. A 4.6 times $(4.6 \times)$ photocurrent enhancement is obtained at the photodetection wavelength of $8.7 \mu \mathrm{m}$. By comparing the simulated diffraction efficiency with the photocurrent enhancement spectrum, we attribute the enhancement to the 1st-order diffraction of the normal infrared (IR) incidence radiation. The photodetectivity $\left(D^{*}\right)$ enhancement at different biases is also measured and analyzed.
\end{abstract}

Keywords: Quantum dot infrared photodetector (QDIP); sub-wavelength surface gratings; broadband photocurrent enhancement.

\section{Introduction}

Quantum dot infrared photodetectors (QDIPs) and focal plane array (FPA) based on intersubband transitions in self-assembled quantum dots (QD) show promising potential for middle wave $(3-5 \mu \mathrm{m})$ infrared (MWIR) and long wave $(8-12 \mu \mathrm{m})$ infrared (LWIR) sensing and imaging. ${ }^{1-9}$ The three-dimensional (3D) quantum confinement structure provides several advantages, including normal incidence photodetection, ${ }^{1,2}$ lower dark current, ${ }^{5}$ high photoreponsitivities, ${ }^{8}$ and high operating temperature. ${ }^{3-5,8}$ However, since the number of active QD layers is limited by the strain and the strain induced defects and dislocations, QDIPs show low quantum efficiencies (QE). Various enhancement techniques have been developed to improve the performance of QDIPs, including: (1) reflection surface grating structures (RSG) ${ }^{7}$; (2) photonic crystal (PC) cavity enhanced QDIP ${ }^{9}$; and (3) surface plasmonic resonance (SPR) enhanced QDIPs. ${ }^{10,11}$ The PC cavity and the SPR enhancement are 
based on resonant effects and are therefore unable to achieve broadband enhancement. ${ }^{9-11}$ In this paper, we report a broadband QDIP enhancement technology based on sub-wavelength transmission surface gratings (TSG). Photocurrent enhancement of 2 to 4.6 times is obtained at a broadband wavelength range from $4-11 \mu \mathrm{m}$. By comparing the simulated diffraction efficiency with the photocurrent enhancement spectrum, we attribute the photocurrent enhancement to the 1st-order diffraction induced coupling of the normal infrared (IR) incidence radiation ( $x$ - or $y$-polarization) to the $z$-polarized IR incidence. The photodetectivity $\left(\mathrm{D}^{*}\right)$ enhancement at different biases is also measured and shows smaller photodetectivity enhancement at higher negative bias levels, suggesting that the high negative bias favors the photocurrent generation with the normal IR incidence.

\section{Device Fabrication}

The QD sample is grown by using a Veeco Gen II MBE system via the StranskiKrastanow (SK) growth mode. A $0.3 \mu \mathrm{m}$ Si-doped $(n+)$ GaAs bottom contact layer $\left(n=1 \times 10^{18} \mathrm{~cm}^{-3}\right)$ is first grown on a semi-insulating GaAs (100) wafer, followed by the growth of a $100 \mathrm{~nm}$ undoped GaAs buffer layer. The growth temperature for the GaAs contact and buffer layers is set to $580^{\circ} \mathrm{C}$. The QD absorption region is then grown. The QDIP absorption region consists of ten-period stacked QD layers. Each QD layer consists of $1 \mathrm{~nm}$ of $\mathrm{In}_{0.15} \mathrm{Ga}_{0.85} \mathrm{As}, 2.4$ monolayer (ML) of InAs QD and $20 \mathrm{ML}$ of $\operatorname{In}_{0.20} \mathrm{Ga}_{0.80} \mathrm{As}$ as capping layers. The QD regions are separated by $65 \mathrm{~nm}$ GaAs spacer layers. The $n$-doping level of the QD region is estimated to be $3.5 \times 10^{17} \mathrm{~cm}^{-3}$, corresponding to 2 electrons doping per QD. The QD layers and cap layers are grown at $\sim 480^{\circ} \mathrm{C}$. The $\mathrm{QD}$ active layer is then covered by a $20 \mathrm{~nm}$ undoped $\mathrm{Al}_{0.20} \mathrm{Ga}_{0.80}$ As top current-blocking layer and $0.1 \mu \mathrm{m}$ highly Si-doped $\left(n=1 \times 10^{18} \mathrm{~cm}^{-3}\right)$ GaAs contact layers. A $1.8 \mu \mathrm{m}$-thick undoped GaAs layer is finally grown on top of the top $n+$ GaAs contacting layer.

After the growth, the wafer is processed into $250 \mu \mathrm{m}$-diameter circular mesas using standard photo-lithography and wet etching procedures. The sub-wavelength surface grating structure is then fabricated on top of the QDIP mesa by using the reactive ion etching (RIE) process. The depth of the sub-wavelength surface gratings is $1.8 \mu \mathrm{m}$. Finally, the top and bottom electrodes are formed by using the E-beam metal evaporation deposition, lift-off and thermal annealing processes. The top and bottom electrodes were conventional N-type Ni/AuGe/Au (150 ̊/300 $/ 4000 \AA)$ alloys. We have verified that the deposited $\mathrm{Ni} / \mathrm{AuGe} / \mathrm{Au}$ layer can effectively cover the top gratings and provide an Ohmic contact to the top contacting layer of the QDIP.

The top view and the zoom-in view of the QDIP with the top sub-wavelength TSG structures are shown in Figs. (1a) and 1(b), respectively. The sub-wavelength TSG structure is a square lattice with a lattice constant $a=4.2 \mu \mathrm{m}$. The width of the square is $d=2.1 \mu \mathrm{m}$. This QDIP with the 4.2- $\mu \mathrm{m}$-period top sub-wavelength TSG is referred to as QDIP-TSG4.2, henceforth. A QDIP without the sub-wavelength TSG structure is also made from the same wafer for the purpose of comparing the performance (referred to as Ref-QDIP, henceforth). 
(a)

(b)

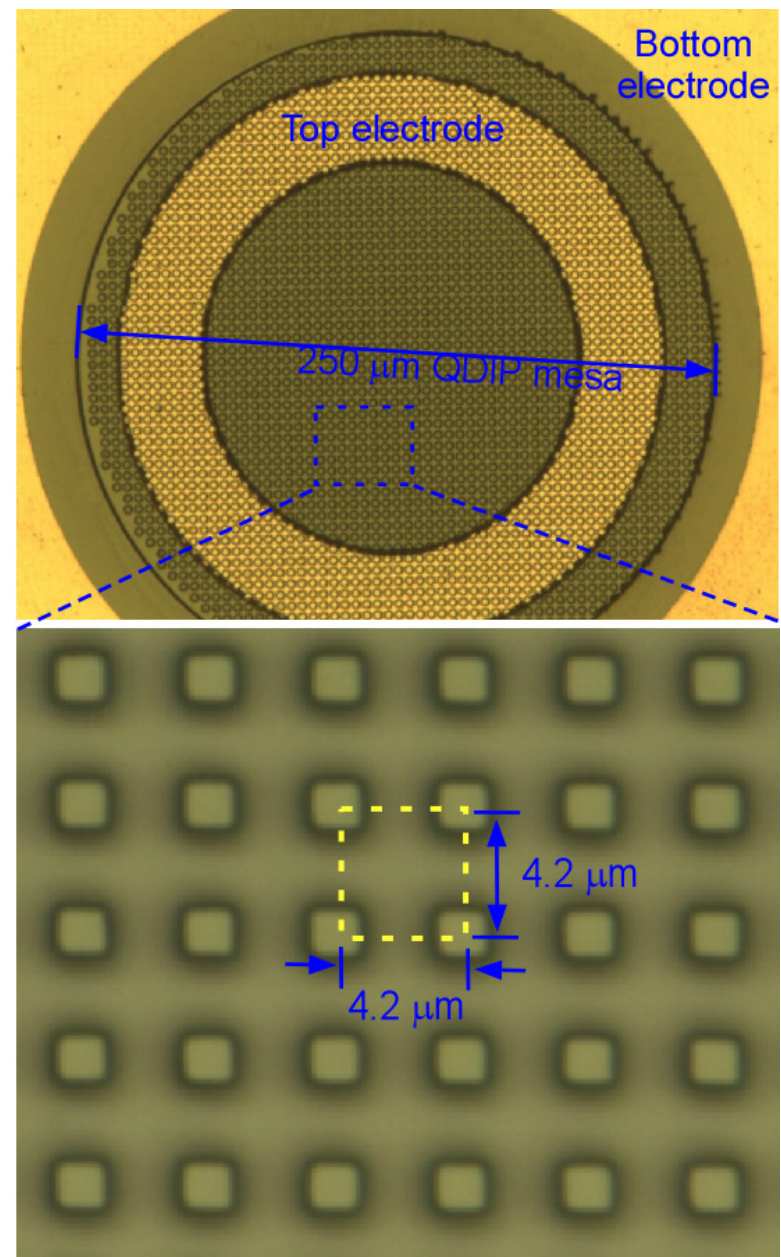

Fig. 1. QDIP with the sub-wavelength TSG. (a) top view; (b) zoom-in view. The 2D sub-wavelength TSG is a square lattice with the lattice constant $a=4.2 \mu \mathrm{m}$. The width of the square is $d=2.1 \mu \mathrm{m}$. The depth of the subwavelength TSG is $1.8 \mu \mathrm{m}$.

\section{Results and Discussions}

Figure 2 shows the photocurrent spectrum of the QDIP-TSG4.2 (upper trace) and the RefQDIP (lower trace) at sample temperature of $77 \mathrm{~K}$ at the bias voltage of $-1.0 \mathrm{~V}$. The QDIPTSG4.2 sample shows a broadband 2-4.6 times photocurrent enhancement from $4 \mu \mathrm{m}$ to $11 \mu \mathrm{m}$. At the wavelength of $8.7 \mu \mathrm{m}$, a 4.6 times photocurrent enhancement is obtained.

To gain better understanding of the photocurrent enhancement, we simulated the 4.2- $\mu \mathrm{m}$ period sub-wavelength TSG structure using Rsoft's DiffractMod ${ }^{\circledR}$. The diffraction efficiencies of different orders of the $4.2-\mu \mathrm{m}$ period TSG structure are shown in Fig. 3. The insets show the configurations of the TSG structure used in the simulation. At long wavelengths from 5-11 $\mu \mathrm{m}$, the 1st-order transmission diffraction efficiency shows a similar trend to the photocurrent enhancement of the QDIP-TSG4.2 sample (Fig. 2). This indicates 


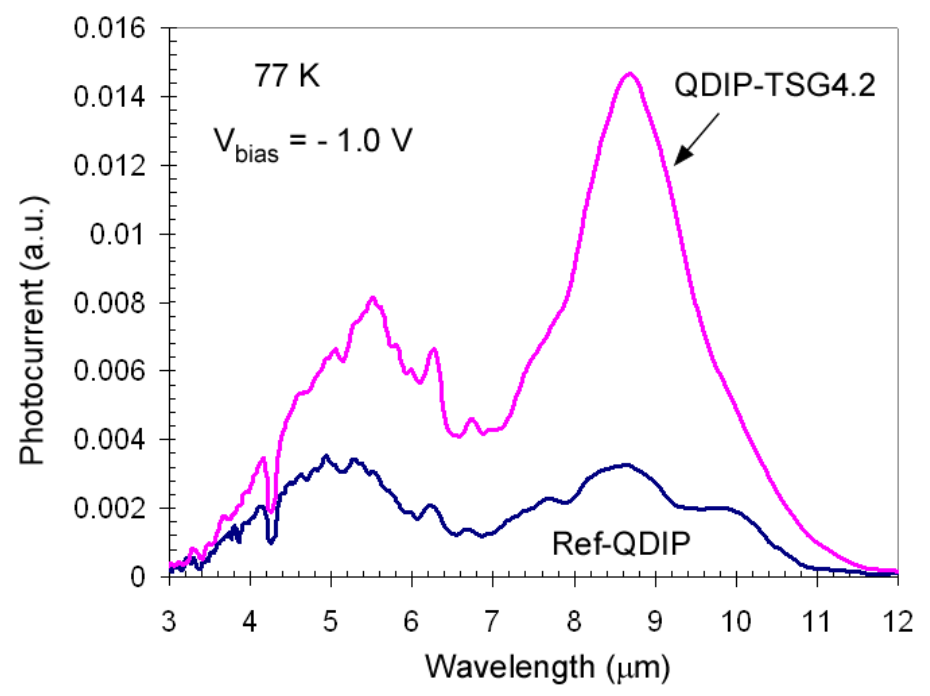

Fig. 2. Photocurrent spectrum of the QDIP-TSG4.2 (upper trace) and the Ref-QDIP (lower trace) at sample temperature of $77 \mathrm{~K}$ at the bias voltage of $-1.0 \mathrm{~V}$. The QDIP-TSG4.2 sample shows a broadband $2-4.6$ times photocurrent enhancement from $4 \mu \mathrm{m}$ to $11 \mu \mathrm{m}$. At the wavelength of $8.7 \mu \mathrm{m}$, a 4.6 times photocurrent enhancement is obtained.

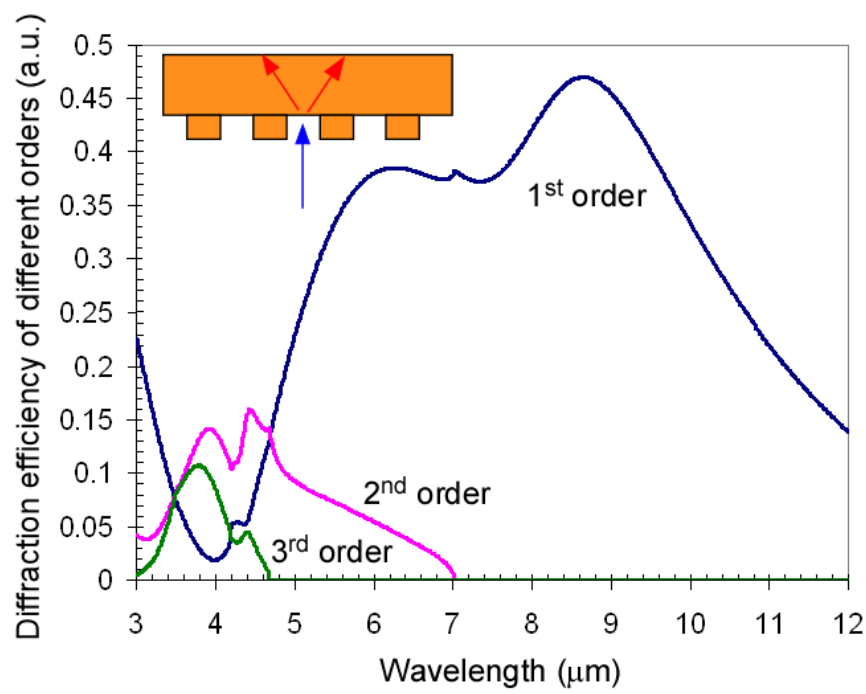

Fig. 3. Diffraction efficiencies of different orders of the 4.2- $\mu \mathrm{m}$ period TSG structure. The insets show the configurations of the TSG structure used in the simulation. At long wavelengths from $5-11 \mu \mathrm{m}$, the 1st-order transmission diffraction efficiency shows a similar trend to the photocurrent enhancement of the QDIP-TSG4.2 sample (Fig. 2). This indicates that the photocurrent enhancement at these wavelengths is primarily due to 1st-order diffraction which couples the normal infrared (IR) incidence radiation ( $x$ - or $y$-polarization) to the $z$-polarized IR incidence. The enhancement at shorter wavelength ranges from $3.5-5 \mu \mathrm{m}$ can be therefore attributed to higher order diffraction. 


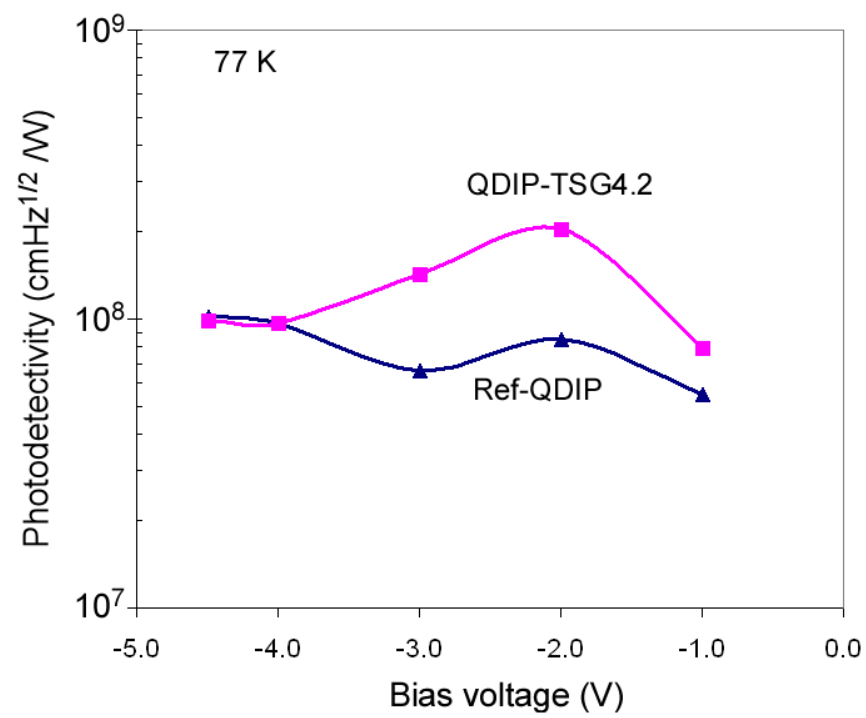

Fig. 4. Photodetectivity D* of the QDIP-TSG4.2 (upper trace) at different bias voltages compared with that of the Ref-QDIP (lower trace) at sample temperature of $77 \mathrm{~K}$. The QDIP-TSG4.2 shows over 2.4 times $(2.4 \times)$ photodetectivity enhancement when the bias is $-2.0 \mathrm{~V}$.

that the photocurrent enhancement at these wavelengths is primarily due to 1st-order diffraction which couples the normal infrared (IR) incidence radiation ( $x$ - or $y$-polarization) to the $z$-polarized IR incidence. The enhancement at shorter wavelength ranges from 3.5-5 $\mu \mathrm{m}$ can be therefore attributed to higher order diffraction.

Figure 4 shows the photodetectivity D* of the QDIP-TSG4.2 (upper trace) at different bias voltages compared with that of the Ref-QDIP (lower trace) at sample temperature of $77 \mathrm{~K}$ (for detailed $\mathrm{D}^{*}$ measurement, please refer to Ref. 8). The QDIP-TSG4.2 shows over 2.4 times $(2.4 \times)$ photodetectivity enhancement when the bias is $-2.0 \mathrm{~V}$. Since the photodetectivity is for the broadband IR signal from $4-11 \mu \mathrm{m}$, the photodetectivity enhancement is smaller than the peak 4.6 times photocurrent enhancement at the wavelength of $8.7 \mu \mathrm{m}$. Note that there is no photodetectivity enhancement at higher negative bias voltages of $-4.0 \mathrm{~V}$ and $-4.5 \mathrm{~V}$. Since the enhancement is due to the coupling of the normal infrared (IR) incidence radiation to the $z$-polarized IR incidence, the smaller photodetectivity enhancement at higher negative bias levels suggests that the high negative bias favors photocurrent generation with the normal IR incidence.

\section{Conclusion}

In conclusion, a long wave infrared quantum dot photodetector with the top subwavelength TSG structure is fabricated. A 2 to 4.6 times broadband photocurrent enhancement is obtained at a broadband wavelength range from $4-11 \mu \mathrm{m}$. The sub-wavelength surface grating structures are simulated and the photocurrent enhancement at long 
wavelengths from $5-11 \mu \mathrm{m}$ is attributed to the 1st-order diffraction induced coupling of the normal infrared (IR) incidence radiation ( $x$ - or $y$-polarization) to the $z$-polarized IR incidence. The photodetectivity $\left(\mathrm{D}^{*}\right)$ enhancement at different biases is also measured and the smaller photodetectivity enhancement at higher negative bias levels suggests that the high negative bias favors photocurrent generation with the normal IR incidence.

\section{Acknowledgment}

This research is partially supported by Air Force Office of Scientific Research (AFOSR) under contract No. FA95501010016. The authors thank Dr. Xifeng Qian of the Photonics center of UML for the MBE growth.

\section{References}

1. H. C. Liu, Quantum dot infrared photodetector, Opto-Electron. Rev. 11 (2003) 1-5.

2. E.-T. Kim, A. Madhukar, Z. Ye and J. C. Campbell, High detectivity InAs quantum-dot infrared photodetectors, Appl. Phys. Lett. 84 (2004) 3277-3279.

3. S. Tsao, H. Lim, W. Zhang and M. Razeghi, High operating temperature $320 \times 256$ middlewavelength infrared focal plane array imaging based on an InAs/InGaAs/InAlAs/InP quantum dot infrared photodetector, Appl. Phys Lett. 90(20) (2007) 201109.

4. P. Bhattacharya, X. H. Su, S. Chakrabarti, G. Ariyawansa and A. G. U. Perera, Characteristics of a tunneling quantum-dot infrared photodetector operating at room temperature, Appl. Phys. Lett. 86 (2005) 191106.

5. L. Jiang, S. S. Li, N. Yeh and J. Chyi, $\mathrm{In}_{0.6} \mathrm{Ga}_{0.4} \mathrm{As} / \mathrm{GaAs}$ quantum-dot infrared photodetector with operating temperature up to $260 \mathrm{~K}$, Appl. Phys. Lett. 82 (2003) 1986-1988.

6. S. Krishna, S. D. Gunapala, S. V. Bandara, C. Hill and D. Z. Ting, Quantum dot based infrared focal plane arrays, Proceed. of the IEEE 95(9) (2007) 1757-1766.

7. S. Gunapala, S. Bandara, C. Hill, D. Ting, J. Liu, Sir B. Rafol, E. Blazejewski, J. Mumolo, S. Keo, S. Krishna, Y. Chang and C. Shott, $640 \times 512$ Pixels long-wavelength infrared (LWIR) quantum-dot infrared photodetector (QDIP) imaging focal plane array, IEEE J. of Quant. Electron. 43(3) (2007) 230-237.

8. X. Lu, J. Vaillancourt and M. J. Meisner, Temperature-dependent photoresponsivity and hightemperature $(190 \mathrm{~K})$ operation of a quantum dot infrared photodetector, Appl. Phys. Lett. 91(5) (2007) 051115.

9. K. T. Posani, V. Tripathi, S. Annamalai, N. R. Weisse-Bernstein, S. Krishna, R. Perahia, O. Crisafulli and O. J. Painter, Nanoscale quantum dot infrared sensors with photonic crystal cavity, Appl. Phys. Lett. 88 (2006) 151104.

10. C.-C. Chang, Y. D. Sharma, Y. S. Kim, J. A. Bur, R. V. Shenoi, S. Krishna, D. Huang and S.-Y. Lin, A surface plasmon enhanced infrared photodetector based on InAs quantum dots, Nano Lett. 10 (2010) 1704-1709.

11. P. Vasinajindakaw, J. Vaillancourt, G. Gu, R. Liu, Y. Ling and X. Lu, A Fano-type interference enhanced quantum dot infrared photodetector, Appl. Phys. Lett. 98 (2011) 211111.

12. R. H. Ritchie, Plasma losses by fast electrons in thin films, Phys. Rev. 106 (1957) 874-881. 